\title{
Demo Abstract: The Signpost Platform for City-Scale Sensing
}

\author{
Joshua Adkins \\ University of California, Berkeley \\ Neal Jackson \\ University of California, Berkeley
}

\author{
Bradford Campbell \\ University of Virginia \\ Pat Pannuto \\ University of California, Berkeley \\ Prabal Dutta \\ University of California, Berkeley
}

\author{
Branden Ghena \\ University of California, Berkeley \\ Samuel Rohrer \\ University of Michigan
}

\begin{abstract}
City-scale sensing holds the promise of enabling deeper insight into how our urban environments function. Applications such as observing air quality and measuring sources of noise pollution can have powerful impacts, allowing city planners and citizen scientists alike to understand and improve their world. However, the path from conceiving applications to implementing them is fraught with many challenges. A successful city-scale deployment requires physical installation, power management, and communications-all challenging tasks standing between a good idea and a realized one, suggesting the need for a platform that enables easy deployment and experimentation of city-scale sensing applications. To address these basic challenges, we present Signpost, a modular platform for city-scale sensing. Signpost simplifies deployment and installation in cities by removing the need for connection to wired infrastructure and instead harvesting energy from an integrated solar panel. The platform provides the key resources necessary for its pluggable sensor modules to support city-scale applications. Signpost stores excess energy for later use, distributes energy between modules, and provides communication through multiple wireless protocols. It also offers local storage for sensor data and allows for additional processing in a duty-cycled Linux environment.
\end{abstract}

\section{CCS CONCEPTS}

- Computer systems organization $\rightarrow$ Embedded systems;

\section{KEYWORDS}

City-Scale Sensing, Signpost, Modular, Platform

\section{ACM Reference Format:}

Joshua Adkins, Bradford Campbell, Branden Ghena, Neal Jackson, Pat Pannuto, Samuel Rohrer, and Prabal Dutta. 2017. Demo Abstract: The Signpost Platform for City-Scale Sensing. In Proceedings of 15th ACM Conference on Embedded Networked Sensor Systems (SenSys'17). ACM, New York, NY, USA, 1 page. https://doi.org/10.1145/3131672.3136990

For questions email adkins@berkeley.edu

Permission to make digital or hard copies of part or all of this work for personal or classroom use is granted without fee provided that copies are not made or distributed for profit or commercial advantage and that copies bear this notice and the full citation on the first page. Copyrights for third-party components of this work must be honored.

For all other uses, contact the owner/author(s).

SenSys'17, November 6-8, 2017, Delft, The Netherlands

(C) 2017 Copyright held by the owner/author(s).

ACM ISBN 978-1-4503-5459-2/17/11.

https://doi.org/10.1145/3131672.3136990
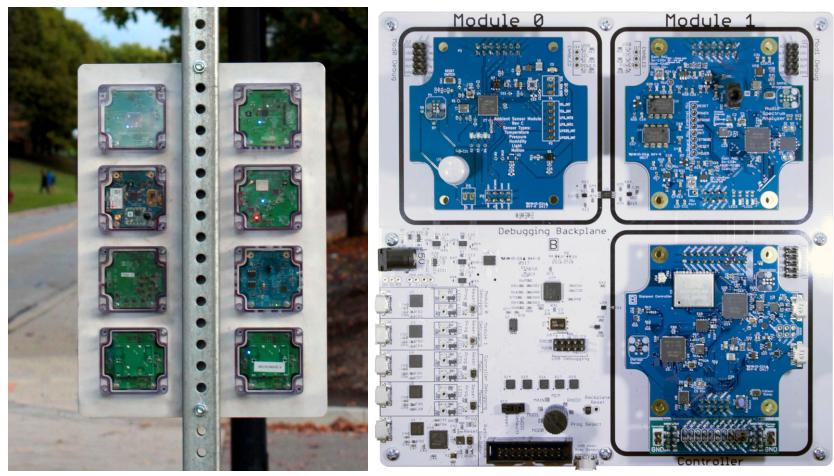

Figure 1: Left: Deployed Signpost. Right: Desktop Development Kit.

\section{DEMO}

This demonstration showcases the newest generation Signpost platform. The demonstrated Signpost will join a network of approximately twenty Signposts deployed around Berkeley, California. Real-time collection of environmental data, privacy-preserving audio-spectrum data, and energy-adaptive policies that autonomously adjust sensor behavior will be demonstrated.

Additionally, a development platform will be demonstrated that showcases the bring-up and local development for new applications and hardware modules. Demo-ers will be able to quickly make modifications (i.e. enabling and disabling sleep) and show how this affects the projected effective duty cycle for a deployed module.

\subsection{Signpost Platform}

The Signpost platform provides reliable energy, networking, storage, and processing to deployed sensor modules. The platform includes robust physical and electrical isolation between modules. System energy is carefully tracked, monitored, and attributed to individual components. Modules can choose from several autonomous energy adaptivity policies, or implement their own, allowing for long term (greater than 24 hour) energy reliability independent of instantaneous harvesting performance.

\subsection{Development Platform}

To facilitate the bring-up of modules before deployment, Signpost introduces a "desktop" development version that eases precise tracing of energy losses as well as more traditional debugging aides, all on a platform designed to support precise mimicry of actual deployed conditions. 